\title{
EXISTENCE AND MULTIPLICITY OF POSITIVE WEAK SOLUTIONS FOR A NEW CLASS OF (P; Q)-LAPLACIAN SYSTEMS
}

\author{
RAFIK GUEFAIFIA, SALAH BOULAARAS, JIABIN ZUO, \\ AND PRAVEEN AGARWAL
}

Received 06 June, 2020

\begin{abstract}
The paper is concerned with the existence of positive weak solutions for a new class of $(p, q)$-Laplacian elliptic systems in a bounded domain by means of the method of sub-super solutions. Particularly, we do not need any sign conditions for $\gamma(0), g(0), f(0)$ and $h(0)$. Moreover, a multiplicity result is obtained when $\gamma(0)=g(0)=f(0)=h(0)=0$. Finally, we give some examples to verify our main results.
\end{abstract}

2010 Mathematics Subject Classification: 35J60; 35B30; 35B40

Keywords: new elliptic systems: existence, positive solutions, multiplicity, sub-super solutions

\section{INTRODUCTION}

In this paper, we deal with the existence and multiplicity of positive weak solutions for the following $(p, q)$-Laplacian systems

$$
\left\{\begin{array}{l}
-\triangle_{p} u-|u|^{p-2} u=\lambda_{1} a(x) f(v)+\mu_{1} \alpha(x) h(u) \text { in } \Omega, \\
-\triangle_{q} v-|v|^{q-2} v=\lambda_{2} b(x) g(u)+\mu_{2} \beta(x) \gamma(v) \text { in } \Omega \\
u=v=0 \text { on } \partial \Omega
\end{array}\right.
$$

where $\triangle_{s} z=\operatorname{div}\left(|\nabla z|^{s-2} \nabla z\right), s>1, \Omega \subset \mathbb{R}^{N}(N \geq 3)$ is a bounded domain with smooth boundary $\partial \Omega, a(x), b(x), \alpha(x), \beta(x) \in C(\bar{\Omega}), \lambda_{1}, \lambda_{2}, \mu_{1}, \mu_{2} \geq 0,1<p, q<$ $\infty$.

The second author would like to thank all the professors of the mathematics department at the University of Annaba in Algeria, especially his Professors/Scientists Pr. Mohamed Haiour, Pr. AhmedSalah Chibi, and Pr. Azzedine Benchettah for the important content of masters and PhD courses in pure and applied mathematics which he received during his studies. Moreover, he thanks them for the additional help they provided to him during office hours in their office about the few concepts/difficulties he had encountered, and he appreciates their talent and dedication for their postgraduate students currently and previously. 
The study of $(p, q)$-Laplacian systems is a new and interesting topic. It arises from electrorheological fluids, nonlinear elasticity theory, etc. (see [5], [15] [1]-[6]). A lot of existence results have been obtained on this class of problems, we refer to ([4], [5], [7], [10], [13], [14], [3], [2], [11], [9], [8]). These problems originate from physical models and are widely used in many fields such as combustion, mathematical biology, chemical reactions and so on. Our method is mainly focused on the method of subsuper solutions (see [10], [11] for a more detailed discussion).

As far as we know, there are very few contributions devoted to the $(p, q)$-Laplacian nonlinear elliptic system. Therefore, with the help of the method of sub-super solutions method, we are inspired by the paper of [12] in which a new $(p, q)$-Laplacian system was discussed and extended our previous results to problem (1.1) without assuming any sign conditions for $h(0), g(0), f(0)$, and $\gamma(0)$. Furthermore, when $h(0)=f(0)=g(0)=\gamma(0)=0$, a multiplicity result is given.

The outline of the paper is organized as follows: Sec. 2 introduces some definitions and make appropriate assumptions, which will be used in the body of the paper. In addition, we show the proof of two important results. Sec. 3, we will illustrate our main results with some interesting examples.

\section{MAIN RESULTS}

First, in order to get our main results, we will consider the following hypothesis: $(H 1)$ There exist $a(x), \alpha(x), b(x), \beta(x) \in C(\bar{\Omega})$ such that

$$
\begin{aligned}
& a(x) \geq a_{1}>0, b(x) \geq b_{1}>0, \\
& \alpha(x) \geq \alpha_{1}>0, \beta(x) \geq \beta_{1}>0 .
\end{aligned}
$$

(H2) Let $f, g, h, \gamma \in C^{1}([0, \infty))$ be monotone functions satisfying

$$
\lim _{s \rightarrow+\infty} f(s)=\lim _{s \rightarrow+\infty} g(s)=\lim _{s \rightarrow+\infty} h(s)=\lim _{s \rightarrow+\infty} \gamma(s)=+\infty .
$$

(H3) $\lim _{s \rightarrow+\infty} \frac{f\left(M(g(s))^{\frac{1}{q-1}}\right)}{s^{p-1}}=0, \forall M>0$.

(H4) $\lim _{s \rightarrow+\infty} \frac{h(s)}{s^{p-1}}=\lim _{s \rightarrow+\infty} \frac{\gamma(s)}{s^{q-1}}=0$.

Next, we define weak solutions and sub-super solutions in $(p, q)$-Laplacian elliptic systems.

Definition 1. Let $(u, v) \in W^{1, p}(\Omega) \cap C(\bar{\Omega}) \times W^{1, q}(\Omega) \cap C(\bar{\Omega})$, we say that $(u, v)$ is a weak solution of problem (1.1), if 


$$
\begin{aligned}
& \int_{\Omega}|\nabla u|^{p-2} \nabla u \cdot \nabla \xi d x-\int_{\Omega}|u|^{p-2} u \cdot \xi d x \\
& =\lambda_{1} \int_{\Omega} a(x) f(v) \xi d x+\mu_{1} \int_{\Omega} \alpha(x) h(u) \xi d x \text { in } \Omega, \\
& \int_{\Omega}|\nabla v|^{q-2} \nabla v \cdot \nabla \zeta d x-\int_{\Omega}|v|^{q-2} v \cdot \zeta d x \\
& =\lambda_{2} \int_{\Omega} b(x) g(u) \zeta d x+\mu_{2} \int_{\Omega} \beta(x) \gamma(v) \zeta d x \text { in } \Omega
\end{aligned}
$$

for all $(\xi, \zeta) \in W_{0}^{1, p}(\Omega) \times W_{0}^{1, q}(\Omega)$.

Definition 2. The nonnegative functions $(\underline{u}, \underline{v}),(\bar{u}, \bar{v})$ in $W^{1, p}(\Omega) \cap C(\bar{\Omega}) \times W^{1, q}(\Omega) \cap$ $C(\bar{\Omega})$ are called a weak subsolution and supersolution of problem (1.1) if they satisfy $(\underline{u}, \underline{v}),(\bar{u}, \bar{v})=(0,0)$ on $\partial \Omega$

$$
\begin{aligned}
& \int_{\Omega}|\nabla \underline{u}|^{p-2} \nabla \underline{u} \cdot \nabla \xi d x-\int_{\Omega}|\underline{u}|^{p-2} \underline{u} \cdot \xi d x \\
& \leq \lambda_{1} \int_{\Omega} a(x) f(\underline{v}) \xi d x+\mu_{1} \int_{\Omega} \alpha(x) h(\underline{u}) \xi d x \text { in } \Omega, \\
& \int_{\Omega}|\nabla \underline{v}|^{q-2} \nabla \underline{v} . \nabla \zeta d x-\int_{\Omega}|\underline{v}|^{q-2} \underline{v} \cdot \zeta d x \\
& \leq \lambda_{2} \int_{\Omega} b(x) g(\underline{u}) \zeta d x+\mu_{2} \int_{\Omega} \beta(x) \gamma(\underline{v}) \zeta d x \text { in } \Omega
\end{aligned}
$$

and

$$
\begin{aligned}
& \int_{\Omega}|\nabla \bar{u}|^{p-2} \nabla \bar{u} \cdot \nabla \xi d x-\int_{\Omega}|\bar{u}|^{p-2} \bar{u} \cdot \xi d x \\
& \geq \lambda_{1} \int_{\Omega} a(x) f(\bar{v}) \xi d x+\mu_{1} \int_{\Omega} \alpha(x) h(\bar{u}) \xi d x \text { in } \Omega, \\
& \int_{\Omega}|\nabla \bar{v}|^{q-2} \nabla \bar{v} \cdot \nabla \zeta d x-\int_{\Omega}|\bar{v}|^{q-2} \bar{v} \cdot \zeta d x \\
& \geq \lambda_{2} \int_{\Omega} b(x) g(\bar{u}) \zeta d x+\mu_{2} \int_{\Omega} \beta(x) \gamma(\bar{v}) \zeta d x \text { in } \Omega
\end{aligned}
$$

for any $(\xi, \zeta) \in W_{0}^{1, p}(\Omega) \times W_{0}^{1, q}(\Omega)$.

In what follows, we shall establish the following the existence result.

Theorem 1. Let $(H 1)-(H 4)$ hold. If $\lambda_{1}+\mu_{1}$ and $\lambda_{2}+\mu_{2}$ are big enough, then problem (1.1) processes a positive weak solution.

Proof. We will show that there exist a positive weak subsolution $(\underline{u}, \underline{v}) \in W^{1, p}(\Omega) \cap$ $C(\bar{\Omega}) \times W^{1, q}(\Omega) \cap C(\bar{\Omega})$ and a supersolution $(\bar{u}, \bar{v}) \in W^{1, p}(\Omega) \cap C(\bar{\Omega}) \times W^{1, q}(\Omega) \cap$ $C(\bar{\Omega})$ of $(1.1)$ such that $\underline{u} \leq \bar{u}, \underline{v} \leq \bar{v}$. Moreover, $(\underline{u}, \underline{v}),(\bar{u}, \bar{v})$ satisfy $(\underline{u}, \underline{v})=(0,0)=$ $(\bar{u}, \bar{v})$ on $\partial \Omega$. 
Let $\sigma_{r}$ be the first eigenvalue of $-\triangle_{r}$, and $\phi_{r}>0$ the corresponding eigenfunction with $\left\|\phi_{r}\right\|=1$ for $r=p, q$. There exist $m, \eta, \delta>0$ such that $\left|\nabla \phi_{r}\right|^{r}-\sigma_{r} \phi_{r} \geq m$ on $\overline{\Omega_{\delta}}=\{x \in \Omega, d(x, \partial \Omega) \leq \delta\}$ and $\phi_{r} \geq \eta$ on $\Omega \backslash \bar{\Omega}_{\delta}$ for $r=p, q$. Taking $k_{0}>0$ such that $a_{1} f(t), \alpha_{1} h(t), b_{1} g(t), \beta_{1} \gamma(t)>-k_{0}$.

First, we claim that

$$
\begin{aligned}
&(\underline{u}, \underline{v}):=\left(\left[\frac{\left(\lambda_{1}+\mu_{1}\right) k_{0}}{m}\right]^{1 / p-1}\left(\frac{p-1}{p}\right) \phi_{p}^{p / p-1}\right. \\
& {\left.\left[\frac{\left(\lambda_{2}+\mu_{2}\right) k_{0}}{m}\right]^{1 / q-1}\left(\frac{q-1}{q}\right) \phi_{q}^{q / q-1}\right) }
\end{aligned}
$$

is a subsolution of problem (1.1) when $\lambda_{1}+\mu_{1}$ and $\lambda_{2}+\mu_{2}$ are big enough. Taking the test function $\xi(x) \in W_{0}^{1, p}(\Omega)$ with $\xi(x) \geq 0$. Thus, from $(H 1)$ we get

$$
\begin{aligned}
& \int_{\Omega}|\nabla \underline{u}|^{p-2} \nabla \underline{u} . \nabla \xi d x-\int_{\Omega}|\underline{u}|^{p-2} \underline{u} . \xi d x \leq \int_{\Omega}|\nabla \underline{u}|^{p-2} \nabla \underline{u} . \nabla \xi d x \\
& =\left(\frac{\left(\lambda_{1}+\mu_{1}\right) k_{0}}{m}\right) \int_{\Omega}\left\{\sigma_{p} \phi_{p}^{p}-\left|\nabla \phi_{p}\right|^{p}\right\} \xi d x \\
& +\left(\frac{\left(\lambda_{1}+\mu_{1}\right) k_{0}}{m}\right) \int_{\Omega \backslash \bar{\Omega}_{\delta}}\left\{\sigma_{p} \phi_{p}^{p}-\left|\nabla \phi_{p}\right|^{p}\right\} \xi d x .
\end{aligned}
$$

We have known that $\left|\nabla \phi_{r}\right|^{r}-\sigma_{r} \phi_{r} \geq m$ for $s=p, q$, on $\overline{\Omega_{\delta}}$. Also on $\Omega \backslash \bar{\Omega}_{\delta} \phi_{r} \geq \eta$ for $r=p, q$. If $\lambda_{1}+\mu_{1}$ and $\lambda_{2}+\mu_{2}$ are big enough in the definition of $\underline{u}, \underline{v}$, then by (H2) we get

$$
a_{1} f(\underline{v}), \alpha_{1} h(\underline{u}), b_{1} g(\underline{u}), \beta_{1} \gamma(\underline{v}) \geq \frac{k_{0}}{m} \max \left\{\sigma_{p}, \sigma_{q}\right\} .
$$

Therefore,

$$
\begin{aligned}
& \int_{\Omega}|\nabla \underline{u}|^{p-2} \nabla \underline{u} . \nabla \xi d x-\int_{\Omega}|\underline{u}|^{p-2} \underline{u} . \xi d x \\
& \leq\left(\frac{\left(\lambda_{1}+\mu_{1}\right) k_{0}}{m}\right) \int_{\Omega_{\delta}}\left\{\sigma_{p} \phi_{p}^{p}-\left|\nabla \phi_{p}\right|^{p}\right\} \xi d x \\
& +\left(\frac{\left(\lambda_{1}+\mu_{1}\right) k_{0}}{m}\right) \int_{\Omega \backslash \bar{\Omega}_{\delta}}\left\{\sigma_{p} \phi_{p}^{p}-\left|\nabla \phi_{p}\right|^{p}\right\} \xi d x \\
& \leq-\left(\lambda_{1}+\mu_{1}\right) k_{0} \int_{\Omega_{\delta}} \xi d x+\left(\frac{\left(\lambda_{1}+\mu_{1}\right) k_{0}}{m}\right) \int_{\Omega \backslash \bar{\Omega}_{\delta}} \sigma_{p} \xi d x \\
& \leq \int_{\Omega_{\delta}}\left[\lambda_{1} a(x) f(\underline{v})+\mu_{1} \alpha(x) h(\underline{u})\right] \xi d x \\
& +\int_{\Omega \backslash \bar{\Omega}_{\delta}}\left[\lambda_{1} a(x) f(\underline{v})+\mu_{1} \alpha(x) h(\underline{u})\right] \xi d x \\
& =\int_{\Omega}\left[\lambda_{1} a(x) f(\underline{v})+\mu_{1} \alpha(x) h(\underline{u})\right] \xi d x
\end{aligned}
$$


Similarly,

$$
\int_{\Omega}|\nabla v|^{q-2} \nabla v \cdot \nabla \zeta d x-\int_{\Omega}|v|^{q-2} v \cdot \zeta d x \leq \int_{\Omega}\left[\lambda_{2} b(x) g(\underline{u})+\mu_{2} \beta(x) \gamma(\underline{v})\right] \zeta d x .
$$

Thus $(\underline{u}, \underline{v})$ is a subsolution of problem (1.1).

Next, let $\omega_{r}$ be a unique positive solution of

$$
\left\{\begin{array}{l}
-\triangle_{r} \omega_{r}=1 \text { in } \Omega \\
\omega_{r}=0 \quad \text { on } \partial \Omega .
\end{array}\right.
$$

for $r=p, q$. We denote

$$
\begin{gathered}
\bar{u}=\frac{C}{v_{p}}\left(\frac{\lambda_{1}\|a\|_{\infty}+\mu_{1}\|\alpha\|_{\infty}}{1-v_{p}^{p-1}}\right)^{\frac{1}{p-1}} \omega_{p} \\
\bar{v}=\left[\left(\frac{\lambda_{2}\|b\|_{\infty}+\mu_{2}\|\beta\|_{\infty}}{1-v_{q}^{q-1}}\right) g\left(C\left(\frac{\lambda_{1}\|a\|_{\infty}+\mu_{1}\|\alpha\|_{\infty}}{1-v_{p}^{p-1}}\right)^{\frac{1}{p-1}}\right)^{\frac{1}{q-1}}\right] \omega_{q}
\end{gathered}
$$

where $v_{r}=\left\|\omega_{r}\right\|_{\infty}, r=p, q$ and $C>0$ is big enough. We claim that $(\bar{u}, \bar{v})$ is a supersolution of $(1.1)$ such that $(\bar{u}, \bar{v}) \geq(\underline{u}, \underline{v})$.

According to $(H 3)-(H 4)$, we can make $C$ big enough so that

$$
\begin{aligned}
& \left(\frac{C}{v_{p}}\right)^{p-1} \geq f\left(\left[\left(\frac{\lambda_{2}\|b\|_{\infty}+\mu_{2}\|\beta\|_{\infty}}{1-v_{q}^{q-1}}\right) g\left(C\left(\frac{\lambda_{1}\|a\|_{\infty}+\mu_{1}\|\alpha\|_{\infty}}{1-v_{p}^{p-1}}\right)^{\frac{1}{p-1}}\right)^{\frac{1}{q-1}}\right] \omega_{q}\right) \\
& +\mu_{1} h\left(\frac{\lambda_{1}\|a\|_{\infty}+\mu_{1}\|\alpha\|_{\infty}}{1-v_{p}^{p-1}}\right)^{\frac{1}{p-1}} \omega_{p} .
\end{aligned}
$$

Hence

$$
\int_{\Omega}|\nabla \bar{u}|^{p-2} \nabla \bar{u} . \nabla \xi d x-\int_{\Omega}|\bar{u}|^{p-2} \bar{u} . \xi d x=\left(\frac{C}{v_{p}}\right)^{p-1}\left(\lambda_{1}\|a\|_{\infty}+\mu_{1}\|\alpha\|_{\infty}\right) \int_{\Omega} \xi d x .
$$


866

RAFIK GUEFAIFIA, SALAH BOULAARAS, JIABIN ZUO, AND PRAVEEN AGARWAL

Using (2.5)

$$
\begin{aligned}
& \int_{\Omega}|\nabla \bar{u}|^{p-2} \nabla \bar{u} . \nabla \xi d x-\int_{\Omega}|\bar{u}|^{p-2} \bar{u} . \xi d x \\
& \geq \lambda_{1}\|a\|_{\infty} f\left(\left[\left(\frac{\lambda_{2}\|b\|_{\infty}+\mu_{2}|| \beta \|_{\infty}}{1-v_{q}^{q-1}}\right) g\left(C\left(\frac{\lambda_{1}\|a\|_{\infty}+\mu_{1}\|\alpha\|_{\infty}}{1-v_{p}^{p-1}}\right)^{\frac{1}{p-1}}\right)^{\frac{1}{q-1}}\right] \omega_{q}\right) \times \\
& \int_{\Omega} \xi d x+\mu_{1}\|\alpha\|_{\infty} \int_{\Omega} h\left(C\left(\frac{\lambda_{1}\|a\|_{\infty}+\mu_{1}\|\alpha\|_{\infty}}{1-v_{p}^{p-1}}\right)^{\frac{1}{p-1}}\right) \xi d x \\
& \geq \int_{\Omega}\left[\lambda_{1} a(x) f(\bar{v})+\mu_{1} \alpha(x) h(\bar{u})\right] \xi d x .
\end{aligned}
$$

Next

$$
\begin{aligned}
& \int_{\Omega}|\nabla \bar{v}|^{q-2} \nabla \bar{v} \cdot \nabla \zeta d x-\int_{\Omega}|\bar{v}|^{q-2} \bar{v} \cdot \zeta d x \\
& =\left\{\left(\lambda_{2}\|b\|_{\infty}+\mu_{2}\|\beta\|_{\infty}\right) g\left(C\left(\frac{\lambda_{1}\|a\|_{\infty}+\mu_{1}\|\alpha\|_{\infty}}{1-v_{p}^{p-1}}\right)^{\frac{1}{p-1}}\right)\right\} \omega_{q} \int_{\Omega} \xi d x \\
& \geq\left[\lambda_{2}\|b\|_{\infty} g\left(C\left(\frac{\lambda_{1}\|a\|_{\infty}+\mu_{1}\|\alpha\|_{\infty}}{1-v_{p}^{p-1}}\right)^{\frac{1}{p-1}}\right)\right. \\
& \left.+\mu_{2}\|\beta\|_{\infty} g\left(C\left(\frac{\lambda_{1}\|a\|_{\infty}+\mu_{1}\|\alpha\|_{\infty}}{1-v_{p}^{p-1}}\right)^{\frac{1}{p-1}}\right)\right] \int_{\Omega} \xi d x .
\end{aligned}
$$

According to $(H 4)$ and choose $C$ big enough, we obtain

$$
\begin{aligned}
& g\left(C\left(\frac{\lambda_{1}\|a\|_{\infty}+\mu_{1}\|\alpha\|_{\infty}}{1-v_{p}^{p-1}}\right)^{\frac{1}{p-1}}\right) \\
& \geq \gamma\left(\left[\left(\frac{\lambda_{2}\|b\|_{\infty}+\mu_{2}\|\beta\|_{\infty}}{1-v_{q}^{q-1}}\right) g\left(C\left(\frac{\lambda_{1}\|a\|_{\infty}+\mu_{1}\|\alpha\|_{\infty}}{1-v_{p}^{p-1}}\right)^{\frac{1}{p-1}}\right)^{\frac{1}{q-1}}\right]\left\|\omega_{q}\right\|_{\infty}\right) .
\end{aligned}
$$


Then from (2.6) we get

$$
\begin{aligned}
& \int_{\Omega}|\nabla \bar{v}|^{q-2} \nabla \bar{v} \cdot \nabla \zeta d x-\int_{\Omega}|\bar{v}|^{q-2} \bar{v} \cdot \zeta d x \\
& \geq \lambda_{2}\|b\|_{\infty} g\left(C\left(\frac{\lambda_{1}\|a\|_{\infty}+\mu_{1}\|\alpha\|_{\infty}}{1-v_{p}^{p-1}}\right)^{\frac{1}{p-1}}\right) \\
& +\mu_{2}\|\beta\|_{\infty} \gamma\left(\left\{\left(\frac{\lambda_{2}\|b\|_{\infty}+\mu_{2}\|\beta\|_{\infty}}{1-v_{q}^{q-1}}\right) g\left(C\left(\frac{\lambda_{1}\|a\|_{\infty}+\mu_{1}\|\alpha\|_{\infty}}{1-v_{p}^{p-1}}\right)^{\frac{1}{p-1}}\right)\right\}^{\frac{1}{q-1}}\left\|\omega_{q}\right\|_{\infty}\right) \\
& \geq \int_{\Omega}\left[b(x) g(\bar{u})+\mu_{2} \beta(x) \gamma(\bar{v})\right] \zeta d x .
\end{aligned}
$$

According to (2.6) and (2.7), we can conclude that $(\bar{u}, \bar{v})$ is a supersolution of (1.1). Further $\bar{u} \geq \underline{u}$ and $\bar{v} \geq \underline{v}$ for $C$ big enough. Thus, we get a solution $(u, v) \in$ $W^{1, p}(\Omega) \cap C(\bar{\Omega}) \times W^{1, q}(\Omega) \cap C(\bar{\Omega})$ of $(1.1)$ with $\underline{u} \leq u \leq \bar{u}$, and $\underline{v} \leq v \leq \bar{v}$. The proof of theorem 1 is complete.

Now we show that the more general system (1.1) possesses at least two distinct positive solutions.

Theorem 2. Suppose that the conditions $(H 1)-(H 4)$ hold. Let $f, g, h$, and $\gamma$ the function be smooth enough around zero with

$$
\begin{aligned}
f(0) & =h(0)=g(0)=\gamma(0)=0 \\
& =f^{(k)}(0)=h^{(k)}(0)=g^{(l)}(0)=\gamma^{(l)}(0)
\end{aligned}
$$

for $k=1,2, \ldots[p-1], l=1,2, \ldots[q-1]$, where $[s]$ denotes the integer part of $s$. Then, problem (1.1) processes at least two positive solutions when $\lambda_{i}+\mu_{i}$ are big enough; $i=1,2$.

Proof. For problem (1.1), we will look for a strict supersolution $\left(\zeta_{1}, \zeta_{2}\right)$, a subsolution $\left(\psi_{1}, \psi_{2}\right)$, a supersolution $\left(z_{1}, z_{2}\right)$, and a strict subsolution $\left(\omega_{1}, \omega_{2}\right)$, such that

$$
\begin{aligned}
& \left(\psi_{1}, \psi_{2}\right) \leq\left(\zeta_{1}, \zeta_{2}\right) \leq\left(z_{1}, z_{2}\right), \\
& \left(\psi_{1}, \psi_{2}\right) \leq\left(\omega_{1}, \omega_{2}\right) \leq\left(z_{1}, z_{2}\right),
\end{aligned}
$$

and $\left(\omega_{1}, \omega_{2}\right) \nless\left(\zeta_{1}, \zeta_{2}\right)$. Then, problem (1.1) processes at least three distinct solutions $\left(u_{i}, v_{i}\right), i=1,2,3$, such that

$$
\begin{aligned}
& \left(u_{1}, v_{1}\right) \in\left[\left(\psi_{1}, \psi_{2}\right),\left(\zeta_{1}, \zeta_{2}\right)\right], \\
& \left(u_{2}, v_{2}\right) \in\left[\left(\omega_{1}, \omega_{2}\right),\left(z_{1}, z_{2}\right)\right]
\end{aligned}
$$

and

$$
\left(u_{3}, v_{3}\right) \in\left[\left(\psi_{1}, \psi_{2}\right),\left(z_{1}, z_{2}\right)\right] \backslash\left(\left[\left(\psi_{1}, \psi_{2}\right),\left(\zeta_{1}, \zeta_{2}\right)\right] \cup\left(\omega_{1}, \omega_{2}\right),\left(z_{1}, z_{2}\right)\right) .
$$

It is obvious that $\left(\psi_{1}, \psi_{2}\right)=(0,0)$ is a (sub)solution. Moreover, we always can find a big supersolution $\left(z_{1}, z_{2}\right)=(\bar{u}, \bar{v})$. Consider 


$$
\left\{\begin{array}{l}
-\triangle_{p} \omega_{1}-\left|\omega_{1}\right|^{p-2} \omega_{1}=\lambda_{1} a(x) \tilde{f}\left(\omega_{2}\right)+\mu_{1} \alpha(x) \tilde{h}\left(\omega_{1}\right) \text { in } \Omega \\
-\triangle_{q} \omega_{2}-\left|\omega_{2}\right|^{q-2} \omega_{2}=\lambda_{2} b(x) \widetilde{g}\left(\omega_{1}\right)+\mu_{2} \beta(x) \widetilde{\gamma}\left(\omega_{2}\right) \text { in } \Omega \\
\omega_{1}=\omega_{2}=0 \text { on } \partial \Omega
\end{array}\right.
$$

where $\widetilde{g}(s)=g(s)-1, \widetilde{\gamma}(s)=\gamma(s)-1, \widetilde{h}(s)=h(s)-1, \widetilde{f}(s)=f(s)-1$. Then by Theorem 1, when $\lambda_{i}+\mu_{i}$ are big enough, we know that the problem (2.10) processes a solution $\left(\omega_{1}, \omega_{2}\right)>0 i=1,2$. It is clear that $\left(\omega_{1}, \omega_{2}\right)$ is a strict subsolution of problem (1.1).

In the end, we will find a strict supersolution $\left(\zeta_{1}, \zeta_{2}\right)$.

Let $\phi_{p}, \phi_{q}$ be the corresponding eigenfunction with respect to operators $\triangle_{p}$ and $\triangle_{q}$ and there exist $C_{1}>0$ and $C_{2}>0$ such that

$$
\phi_{p} \leq C_{1} \phi_{q} \text { and } \phi_{q} \leq C_{2} \phi_{p}
$$

Let $\left(\zeta_{1}, \zeta_{2}\right)=\left(\rho \phi_{p}, \rho \phi_{q}\right)$, where $\rho>0$,

$$
G_{p}(x):=\left(\sigma_{p}-1\right) x^{p-1}-\lambda_{1} f\left(C_{2} x\right)-\mu_{1} h(x)
$$

and

$$
G_{q}(x):=\left(\sigma_{q}-1\right) x^{q-1}-\lambda_{2} g\left(C_{1} x\right)-\mu_{2} \gamma(x) .
$$

Note that $G_{p}(0)=G_{q}(0)=0, G_{p}^{(k)}(0)=G_{q}^{(l)}(0)=0$ for $k=1,2, \ldots[p-1]$ and $l=1,2, \ldots .[q-1]$.

$$
\left\{\begin{array}{l}
G_{p}^{(p-1)}(0)>0 \text { and } G_{q}^{(q-1)}(0)>0 \text { if } p, q \in \mathbb{Z}^{+}, \\
\lim _{r \rightarrow+\infty} G_{p}^{([p])}(r)=+\infty=\lim _{r \rightarrow+\infty} G_{p}^{([q])}(r) \text { if } p, q \notin \mathbb{Z}^{+} .
\end{array}\right.
$$

Hence, there exists $\theta$ such that $G_{q}(x)>0$ and $G_{p}(x)>0$ for any $x \in(0, \theta]$. So, for $0<\rho \leq \theta$ we get

$$
\left(\sigma_{p}-1\right) \zeta_{1}^{p-1}=\left(\sigma_{p}-1\right)\left(\rho \phi_{p}\right)^{p-1}>\lambda_{1} f\left(C_{2} \rho \phi_{p}\right)-\mu_{1} h\left(\rho \phi_{p}\right) .
$$

By (2.10) and the monotonicity of function $f$, we obtain

$$
\begin{aligned}
& \left(\sigma_{p}-1\right) \zeta_{1}^{p-1}=\left(\sigma_{p}-1\right)\left(\rho \phi_{p}\right)^{p-1} \\
& >\lambda_{1} f\left(C_{2} \rho \phi_{p}\right)-\mu_{1} h\left(\rho \phi_{p}\right) \\
& \geq \lambda_{1} f\left(\rho \phi_{q}\right)-\mu_{1} h\left(\rho \phi_{p}\right)=\lambda_{1} f\left(\zeta_{2}\right)-\mu_{1} h\left(\zeta_{1}\right)
\end{aligned}
$$

for any $x \in \Omega$. In the same way, we also have

$$
\begin{aligned}
& \left(\sigma_{q}-1\right) \zeta_{2}^{q-1}=\left(\sigma_{q}-1\right)\left(\rho \phi_{q}\right)^{q-1} \\
& >\lambda_{2} g\left(C_{1} \rho \phi_{q}\right)-\mu_{2} \gamma\left(\rho \phi_{q}\right) \\
& \geq \lambda_{2} g\left(\rho \phi_{p}\right)-\mu_{2} \gamma\left(\rho \phi_{q}\right)=\lambda_{2} g\left(\zeta_{1}\right)-\mu_{2} \gamma\left(\zeta_{2}\right),
\end{aligned}
$$


for any $x \in \Omega$. Making use of (2.11) and (2.12), we obtain

$$
\begin{aligned}
& \int_{\Omega}\left|\nabla \zeta_{1}\right|^{p-2} \nabla \zeta_{1} \cdot \nabla \xi d x-\int_{\Omega}\left|\zeta_{1}\right|^{p-2} \zeta_{1} \cdot \xi d x \\
& =\rho^{p-1}\left\{\int_{\Omega}\left|\nabla \phi_{p}\right|^{p-2} \nabla \phi_{p} . \nabla \xi d x-\int_{\Omega}\left|\phi_{p}\right|^{p-2} \phi_{p} \cdot \xi d x\right\} \\
& =\int_{\Omega}\left\{\sigma_{p}\left(\rho \phi_{p}\right)^{p-1}-\left(\rho \phi_{p}\right)^{p-1}\right\} \xi d x .
\end{aligned}
$$

Since because $\phi_{p}>0$, we have

$$
\begin{aligned}
& \int_{\Omega}\left\{\sigma_{p}\left(\rho \phi_{p}\right)^{p-1}-\left(\rho \phi_{p}\right)^{p-1}\right\} \xi d x \\
& =\int_{\Omega}\left\{\left(\sigma_{p}-1\right)\left(\rho \phi_{p}\right)^{p-1}\right\} \xi d x \\
& >\lambda_{1} \int_{\Omega} f\left(\zeta_{2}\right) \xi d x-\mu_{1} \int_{\Omega} h\left(\zeta_{1}\right) \cdot \xi d x,
\end{aligned}
$$

Similarly we also have

$$
\int_{\Omega}\left|\nabla \zeta_{2}\right|^{q-2} \nabla \zeta_{2} \cdot \nabla \xi d x-\int_{\Omega}\left|\zeta_{2}\right|^{q-2} \zeta_{2} \cdot \xi d x>\lambda_{2} \int_{\Omega} g\left(\zeta_{1}\right) \xi d x-\mu_{2} \int_{\Omega} \gamma\left(\zeta_{2}\right) \xi d x .
$$

It follows that $\left(\zeta_{1}, \zeta_{2}\right)$ is a strict supersolution. Let $\rho$ small enough so that $\left(\omega_{1}, \omega_{2}\right) \nless$ $\left(\zeta_{1}, \zeta_{2}\right)$. So, we can find solutions

$$
\left(u_{1}, v_{1}\right) \in\left[\left(\psi_{1}, \psi_{2}\right),\left(\zeta_{1}, \zeta_{2}\right)\right],\left(u_{2}, v_{2}\right) \in\left[\left(\omega_{1}, \omega_{2}\right),\left(z_{1}, z_{2}\right)\right]
$$

and

$$
\left(u_{3}, v_{3}\right) \in\left[\left(\psi_{1}, \psi_{2}\right),\left(z_{1}, z_{2}\right)\right] \backslash\left(\left[\left(\psi_{1}, \psi_{2}\right),\left(\zeta_{1}, \zeta_{2}\right)\right] \cup\left(\omega_{1}, \omega_{2}\right),\left(z_{1}, z_{2}\right)\right) .
$$

This fact that $\left(u_{1}, v_{1}\right) \equiv\left(\psi_{1}, \psi_{2}\right) \equiv(0,0)$ can happen due to $\left(\psi_{1}, \psi_{2}\right) \equiv(0,0)$ is a solution. So, anyway we can find two positive solutions $\left(u_{2}, v_{2}\right)$ and $\left(u_{3}, v_{3}\right)$. Therefore, we conclude the proof of Theorem2.

\section{EXAMPLES}

Example 1. Let

$$
\begin{aligned}
& f(x)=\sum_{i=1}^{m} a_{i} x^{p_{i}}-c_{1}, g(x)=\sum_{j=1}^{n} b_{j} x^{q j}-c_{2} \\
& h(x)=\sum_{k=1}^{s} \alpha_{k} x^{r_{k}}-c_{3}, \gamma(x)=\sum_{l=1}^{\tau} \beta_{l} x^{d_{l}}-c_{4},
\end{aligned}
$$

where

$$
d_{j}<(q-1), r_{k}<(p-1), p_{i} q_{j}<(p-1)(q-1)
$$

and

$$
a_{i}, b_{j}, \alpha_{k}, \beta_{l}, p_{i}, q_{j}, r_{k}, d_{j}, c_{1}, c_{2}, c_{3}, c_{4} \geq 0
$$


So, it is clear that $f, g, h$ and $\gamma$ fulfill the assumptions of Theorem 1.

Example 2. Let

$$
\begin{aligned}
& f(x)=\left\{\begin{array}{l}
x^{p_{1}}, x \leq 1, \\
\frac{p_{1}}{p_{2}} x^{p_{2}}+\left(1-\frac{p_{1}}{p_{2}}\right), x>1,
\end{array}, h(x)=\left\{\begin{array}{l}
x^{p_{3}}, x \leq 1, \\
\frac{p_{3}}{p_{4}} x^{p_{4}}+\left(1-\frac{p_{3}}{p_{4}}\right), x>1,
\end{array}\right.\right. \\
& g(x)=\left\{\begin{array}{l}
x^{q_{1}}, x \leq 1, \\
\frac{q_{1}}{q_{2}} x^{q_{2}}+\left(1-\frac{q_{1}}{q_{2}}\right), x>1,
\end{array}, \gamma(x)=\left\{\begin{array}{l}
x^{q_{3}}, x \leq 1, \\
\frac{q_{3}}{q_{4}} x^{q_{4}}+\left(1-\frac{q_{3}}{q_{4}}\right), x>1,
\end{array}\right.\right.
\end{aligned}
$$

where we suppose that

$$
\left\{\begin{array}{l}
p_{1}, p_{3}>p-1 \text { if } p \in \mathbb{Z}^{+} \\
p_{1}, p_{3}>[p] \text { if } p \notin \mathbb{Z}^{+} \\
q_{1}, q_{3}>q-1 \text { if } q \in \mathbb{Z}^{+} \\
q_{1}, q_{3}>[q] \text { if } q \notin \mathbb{Z}^{+}
\end{array}\right.
$$

$p_{4}<p-1, p_{2} q_{2}<(p-1)(q-1)$ and $q_{4}<q-1$. Clearly, $f, g, h$ and $\gamma$ fulfill all the assumptions of Theorem 2

\section{ACKNOWLEDGEMENT}

The third author was supported by the Fundamental Research Funds for Central Universities (2019B44914) and the National Key Research and Development Program of China (2018YFC1508100), the China Scholarship Council (201906710004).

\section{AVAILABILITY OF DATA AND MATERIALS}

Not applicable.

\section{COMPETING INTERESTS}

The authors declare that there is no conflict of interests regarding the publication of this manuscript. The authors declare that they have no competing interests.

\section{AUTHORS' CONTRIBUTIONS}

The authors contributed equally in this article. They have all read and approved the final manuscript.

\section{REFERENCES}

[1] G. A. Afrouzi and K. J. Brown, "Positive solutions for a semilinear elliptic problem with a signchanging nonlinearity." Nonlinear Anal., vol. 36, no. 4, pp. 507-510, 1999, doi: 10.1016/S0362.

[2] G. A. Afrouzi, S. Shakeri, and N. T. Chung, "Existence of positive solutions for variable exponent elliptic systems with multiple parameters." Afr. Mat., vol. 26, pp. 159-168, 2015, doi: 10.1007/s13370-013-0196-9. 
[3] M. Alizadeh and M. Alimohammady, "Regularity and entropy solutions of some elliptic equations." Miskolc Mathematical Notes., vol. 19, no. 2, pp. 715-729, 2018, doi: 10.18514/MMN.2018.2545.

[4] S. Boulaaras, "Some existence results for elliptic Kirchhoff equation with changing sign data and a logarithmic nonlinearity." Journal of Intelligent and Fuzzy Systems, in press., vol. 37, no. 6, pp. 8335 -8344, 2019, doi: 10.3233/JIFS-190885.

[5] S. Boulaaras and R. Guefaifia, "Existence of positive weak solutions for a class of Kirrchoff elliptic systems with multiple parameters." Mathematical Methods in the Applied Sciences., vol. 41, no. 13, pp. $5203-5210,2018$, doi: 10.1002/mma.5071.

[6] Y. Chen, S. Levine, and M. Rao, "Variable exponent, linear growth functionals in image restoration." SIAM J. Appl. Math., vol. 66, no. 4, pp. 1383 -1406, 2006, doi: 10.1137/050624522.

[7] N. T. Chung, "Multiple solutions for a $\mathrm{p}(\mathrm{x})$-Kirchhoff-type equation with sign-changing nonlinearities." Complex Variables and Elliptic Equations., vol. 58, no. 12, pp. 1637 -1646, 2013, doi: 10.1080/17476933.2012.701289.

[8] S. Gala, Q. Liu, and M. A. Ragusa, "A new regularity criterion for the nematic liquid crystal fows." Applicable Analysis., vol. 91, no. 9, pp. 1741 -1747, 2012, doi: 10.1080/00036811.2011.581233.

[9] S. Gala and M. A. Ragusa, "Logarithmically improved regularity criterion for the Boussinesq equations in Besov spaces with negative indices." Applicable Analysis., vol. 95, no. 6, pp. 12711279, 2016, doi: https://doi.org/10.1080/00036811.2015.1061122.

[10] R. Guefaifia and S. Boulaaras, "Existence of positive solution for a class of (p(x),q(x))-Laplacian systems." Rend. Circ. Mat. Palermo., vol. 67, pp. 93 -103, 2018, doi: 10.1007/s12215-017-02977.

[11] R. Guefaifia and S. Boulaaras, "Sub-super solutions method for elliptic systems involving $\left(p_{1}, \ldots, p_{m}\right)$ - Laplacian operator." Mathematical methods in the applied sciences., vol. 43, no. 7, pp. $4191-4199$, 2020, doi: 10.1002/mma.6183.

[12] R. Guefaifia, S. Boulaaras, S. Alodhaibi, and S. Alkhalaf, "Existence of positive weak solutions for a new class of $(\mathrm{p}, \mathrm{q})$ Laplacian nonlinear elliptic system with sign-changing weights." Complexity., vol. 2020, no. 2020, pp. 1-9, 2020, doi: 10.1155/2020/5302513.

[13] S. Polidoro and M. A. Ragusa, "Harnack inequality for hypoelliptic ultraparabolic equa- tions with a singular lower order term." Revista Matematica Iberoamericana., vol. 24, no. 3, pp. 1011 -1046, 2008, doi: 10.1002/mma.6183.

[14] Q. H. Zhang, "Existence of positive solutions for a class of $\mathrm{p}(\mathrm{x})$-Laplacian systems." J. Math. Anal. Appl., vol. 333, pp. 591 -603, 2007, doi: 10.1016/j.jmaa.2006.11.037.

[15] V. V. Zhikov, "Averaging of functionals of the calculus of variations and elasticity theory." Math. USSR., vol. 29, no. 1, pp. 33-36, 1987.

Authors' addresses

Rafik Guefaifia

Department of Mathematics, Faculty of Exact Sciences,, University Tebessa, Tebessa, Algeria, 12000 Tebessa, Algeria

E-mail address: rafikik982@gmail.com

Salah Boulaaras

Department of Mathematics, College of Sciences and Arts in Al-Rass, Qassim University, Buraidah, 51452 Buraidah, Saudi Arabia

Laboratory of Fundamental and Applied Mathematics of Oran (LMFAO),, University of Oran 1, Oran, 31000 Oran, Algeria

E-mail address: s.boularas@qu.edu.sa 
Jiabin Zuo

Faculty of Applied Sciences, Jilin Engineering Normal University,, 130052, Changchun, China

College of Science, Hohai University,, 210098, Nanjing, China

Departamento de Matemática, Universidade Estadual de Campinas,, 13083-859, IMECC, Campinas, SP CEP, Brazil

E-mail address: zuojiabin88@163.com

\section{Praveen Agarwal}

Anand International College of Engineering, Near Kanota, Agra Road,, 303012 Rajasthan, India

E-mail address: goyal.praveen2011@gmail.com 